\title{
LA CIUDAD EN LA INFORMACIÓN RADIOFÓNICA LOCAL
}

\author{
Laura Teruel Rodríguez
}

(Universidad de Málaga)

teruel@uma.es

\section{Resumen:}

En el actual panorama mediático, en constante evolución, la información local es un referente primario para la ciudadanía. El periodismo de proximidad goza de una cercanía privilegiada con su audiencia que debe traducirse en unos contenidos que dinamicen el debate social en torno a unos valores y escenarios compartidos. El presente trabajo se centra en el estudio del periodismo local, más profundamente sobre política, que desarrolla la radio en Málaga. Este texto analiza la imagen que se construye de una ciudad que se encuentra en un momento de gran desarrollo a través de una emisora pública y otra privada.

Palabras claves: Radio, periodismo local, Málaga, contenidos, política, agenda.

\begin{abstract}
:
In the current media panorama, in constant evolution, the local information is a primary frame for citizenship. The local journalism enjoys a privileged nearness with his audience and it must involve that his contents activate the social debate about shared values and questions. The present papers are focused on the study of the political local journalism developed by the radio in the city of Malaga. This text analyzes the image that is composed of that city, which is living a moment of great development, across a public station and another private one.
\end{abstract}

Key words: Radio, local journalism, Malaga, contents, policy, agenda.

\section{INTRODUCCIÓN}

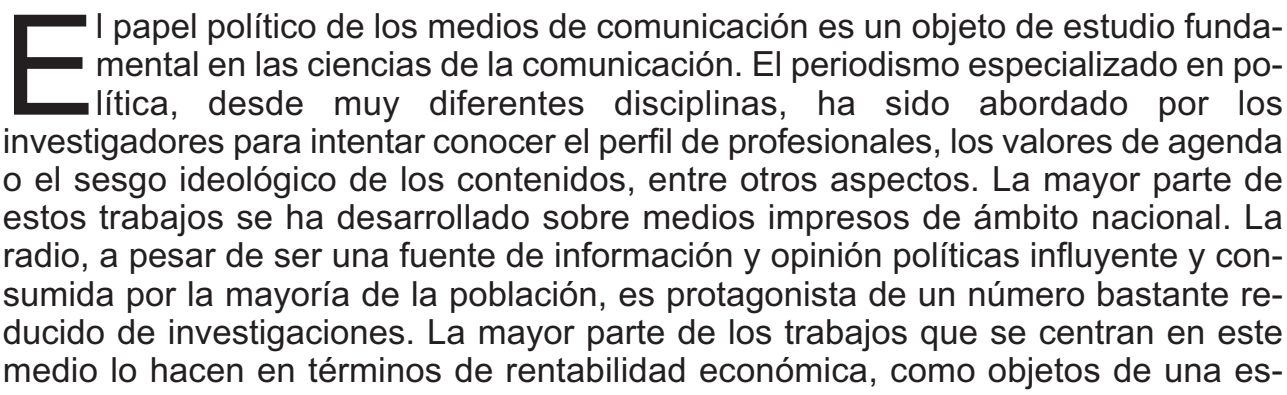


tructura empresarial mayor. Se estudia, por ejemplo, el proceso de digitalización de la radio en España o la repercusión de los medios digitales en las cadenas (Gutiérrez y Huertas, 2003). La volatilidad de las emisiones, frente al editable soporte digital o el perenne referente en papel, dificulta también el acceso a éstas.

La fugacidad e inmediatez de sus contenidos junto a la dificultad añadida que suponen las desconexiones locales o autonómicas para realizar un análisis de la parrilla radiofónica, ha supuesto que este medio, eterno superviviente a los avances tecnológicos y con una audiencia estable, haya sido menos analizado. Por eso, en un periodo de cambios como el actual, la radio necesita especialmente de estudios que aborden cuál ha de ser el proceso de adaptación, cuál es su valor añadido y en qué frentes puede competir con el resto de medios.

Actualmente, la radio, como los restantes soportes mediáticos, se encuentra inmersa en un proceso en el que el cambio se ha convertido en un elemento estructural. Esto es, lejos de encaminarse a encontrar un estadio de convivencia ideal entre todos los medios, la capacidad de transformarse se ha convertido en una cualidad imprescindible de éstos porque el panorama evoluciona constantemente.

\begin{abstract}
Y en la última mitad del siglo XX lo hacen estableciendo una doble y simultánea tendencia global-local. Esta fase de grandes transformaciones, que camina de la mano de las tecnologías actuales y de la economía, nos ha conducido a una sociedad más mundializada en la que se produce una revalorización y un redescubrimiento de lo local. En la actualidad, global y local constituyen las dos caras de una misma moneda, la que simboliza el hipersector de la comunicación. Y, mientras avanza la globalización o se multiplican las redes que facilitan la intercomunicación en tiempo real, el valor social de la información de proximidad también aumenta (López García, 2000).
\end{abstract}

La necesidad de efectuar investigaciones sobre la radio y el periodismo de proximidad en este escenario impulsa este trabajo. Más aún en tanto, en el panorama actual, frente a la homogeneización que imponen los grandes entramados mediáticos, los ciudadanos demuestran que siguen valorando el carácter local de las noticias y la información (Merli, 2000). La información de carácter local sigue siendo la que más impacto tiene entre los habitantes de un mismo entorno y, también, sobre el tejido socioeconómico de dicho lugar. Si bien los avances tecnológicos en los emisores nacionales e internacionales hicieron temer a los medios locales que podrían perder su protagonismo, lo cierto es que la cercanía privilegiada con sus destinatarios es un valor añadido que los medios locales han sabido aprovechar para consolidarse como interlocutores imprescindibles de su comunidad.

Para su pervivencia, para seguir ocupando un papel trascendente en su ámbito de actuación, el periodismo de proximidad debe ser consciente de que su principal valía reside en sus contenidos, en su capacidad para retroalimentarse con la sociedad que lo alberga. El periodismo local es el que encuentra menos distancias para dar cabida a las voces de los oídos que le escuchan; esto es, ha de coadyuvar al establecimiento de una democracia comunicativa, con pluralidad de emisores. Pues, si los miembros de la comunidad no tienen cabida en la agenda de un medio local, si sus contenidos se reducen a ámbitos políticos o a tratar temas que también abordan los medios autonómicos o nacionales, la audiencia se decantará por éstos: 
Un medio local existe en relación con un espacio social, con el que mantiene una relación de retroalimentación mutua. El público se reconoce como identificado, se ve reflejado como si estuviese ante un espejo; esa imagen reflejada responde al deseo de protagonismo de cada uno de los usuarios. (López et ali, 1998).

Entendiendo la política en su sentido más amplio, la información de proximidad es una mano tendida para la participación ciudadana. Frente a la uniformada oferta informativa del resto de medios, en las emisoras locales los ciudadanos y colectivos sociales encuentran el debate sobre lo inmediato, sobre lo cercano, y ello les anima a participar. Si el debate contempla las señas de identidad locales y permite el acceso a la pluralidad de actores ciudadanos, se convierte en un contrafuerte de la información nacional, en unas primeras coordenadas a través de las cuales los ciudadanos entienden el mundo.

Pero, de un tiempo a esta parte, aunque especialmente en la última legislatura, numerosos autores vienen advirtiendo que la información radiofónica está sufriendo especialmente el proceso de politización mediática (Rodero y Sánchez, 2007). El proceso de politización está llevando a una pérdida de credibilidad del medio radiofónico que es especialmente acuciante en la radio local porque, en primer lugar, ésta casi no existe. Lo que se analiza en este texto son los contenidos locales de la radio nacional generalista, las desconexiones locales de emisoras públicas y privadas; puesto que la existencia de radios netamente malagueñas es prácticamente nula. En este sentido, Rodero y Sánchez se plantean que existe un escaso interés empresarial por la radio local y que, además, ésta se encuentra especialmente indefensa ante el proceso de polarización o politización mediática.

Por ello, este trabajo estudia el papel de la información política en la radio local. Se analiza, especialmente, la cobertura política de los temas locales. Teniendo en cuenta los objetivos trazados por López, Galindo y Villar para la pervivencia de la radio local, el presente trabajo observa si estos medios ayudan a consolidar la democracia comunicativa y facilitar el acceso de los receptores actores al escenario mediático (López et ali, 1998) y, por tanto, respetando la diversidad, se convierten en un foro para lo más próximo y, con ello, fomentan el interés y la participación ciudadana.

\section{LAS COORDENADAS DEL ESTUDIO: LA CIUDAD Y LAS CADENAS DE RADIO}

\subsection{La ciudad: Málaga}

Málaga es una ciudad en crecimiento. Según los indicadores de la Fundación CIEDES para el periodo analizado, la provincia, a pesar de la ralentización de la economía nacional, seguía creciendo por encima de la media española. Y ello ha sucedido durante los últimos años en los que ha sido, además, la provincia andaluza más dinámica (Blanco, 2007).

El futuro se perfila igualmente esperanzador. La ciudad es la cuarta en los presupuestos generales del Estado para 2008, con una partida de más de 1.000 millones, 
y hay numerosas infraestructuras en desarrollo que cambiarán la fisonomía y la vida cotidiana de la ciudad. El AVE a Madrid, inaugurado el 23 de diciembre de 2007, ha sido el primer gran proyecto en ver la luz pero se espera que, durante la siguiente legislatura, queden prácticamente listos las dos líneas de metro, la segunda pista del aeropuerto y la nueva terminal, la hiperronda, la autopista de Las Pedrizas, la ampliación del Parque Tecnológico de Andalucía, el nuevo hospital de la ciudad, la autovía entre Antequera y Córdoba, la rehabilitación del mercado de Atarazanas, el corredor ferroviario de la Costa del Sol, y el nuevo puerto, entre otros. La entidad de algunas de estas infraestructuras, aisladamente, haría pensar que ésta es una ciudad que se encuentra en una fase de desarrollo. Pero la conjunción de todas ellas en el mismo escenario, que crece sistemáticamente en número de habitantes, presenta un panorama de cambio incuestionable. La fisonomía de la ciudad va a progresar tanto en los próximos años que variará inexorablemente el concepto que los ciudadanos tienen de Málaga.

Pero la agenda mediática malagueña encuentra otro gran asunto en la capital y es meramente político. El consistorio es gobernado por el Partido Popular, con mayoría absoluta desde hace varias legislaturas. La Junta de Andalucía tiene signo socialista, al igual que la Diputación de Málaga y el Gobierno. En un escenario como el que se ha dibujado, con numerosos proyectos que exigen la colaboración y el acuerdo entre administraciones, las discrepancias son públicas ${ }^{1}$. Tanto es así que incluso el director de Metro Málaga, Enrique Urkijo, ha declarado que el enfrentamiento institucional en el País Vasco era menor de lo que se viene encontrando en Málaga desde su llegada ${ }^{2}$.

\subsection{Las emisoras de radio}

Este análisis se basa en los informativos locales de Canal Sur Radio, una emisora pública de ámbito autonómico, y Ser Málaga, las desconexiones locales de cadena privada con más audiencia del país desde hace 13 años ${ }^{3}$.

La cadena Ser celebra este año su $25^{\circ}$ aniversario en la ciudad. En 1982 se instaló en Málaga la emisora local y, actualmente, es una de las más escuchadas por los malagueños. Forma parte de la red de emisoras de radio del Grupo PRISA junto con las musicales 40 Principales, Cadena Dial, M80 Radio, Máxima FM y Radiolé. No sólo por la audiencia de sus programas nacionales sino también por su larga tradición y relevancia en la ciudad, es incuestionable que es una de las emisoras más

\footnotetext{
${ }^{1}$ Estas discrepancias han sido especialmente visibles en algunos asuntos como la inauguración del nuevo Palacio de Justicia, la aprobación del PGOU o la llegada del Ave a la ciudad. Este último caso es muy elocuente: se organizaron dos fiestas paralelas, en el mismo sitio y a la misma hora, una presidida por el presidente del Gobierno y la ministra de Fomento, a la que fue invitado el alcalde, y otra organizada por éste y su equipo para los ciudadanos. Ambas estaban separadas tan sólo por una pared.

2 Disponible en http://www.diariosur.es/pg060611/prensa/noticias/Portada/200606/11/SUR-POR261.html [Consulta: 20 de enero de 2008].

${ }^{3}$ Véase "La Cadena Ser, líder de la radio española con 4.643 .000 oyentes". Disponible en: http://www.elpais.com/articulo/sociedad/Cadena/Ser/lider/radio/espanola/4643000/oyentes/elpepu soc/20070703elpepusoc_1/Tes [Consulta: 28 de noviembre de 2007].
} 
importantes para los malagueños. Existen emisoras de la Ser en Ronda, Estepona y Vélez Málaga pero la redacción de la capital dice que a Marbella llega la señal de Málaga. Por tanto, hay que considerar que, en la cobertura de la provincia, estas comarcas son preferentemente tratadas en sus emisoras de cabecera ${ }^{4}$.

La radio autonómica andaluza comenzó sus emisiones al final de 1988. Junto con Canal Sur Televisión, esta emisora despertó un gran interés en la audiencia andaluza que podía disfrutar así de sus únicos medios de ámbito autonómico, ya que no existen periódicos para toda la comunidad andaluza. Afirma tener una parrilla que combina entretenimiento con información, y que presta atención especial a la participación de los oyentes. A nivel andaluz, es uno de los medios de radiodifusión líder. El grupo de Radio Televisión Andaluza cuenta, además, con Canal Fiesta Radio y Radio Andalucía Información.

No realiza desconexiones comarcales. En una entrevista telefónica, se preguntó a los redactores de informativos sobre sus líneas temáticas preferentes, si primaba la información de la ciudad o de la provincia. La opinión de uno de los periodistas de Canal Sur fue la siguiente: olvídate de la capital, los temas que cubrimos son también de los pueblos. Aunque la capital es la que más información genera, más temas políticos, de la Junta de Andalucía, del Gobierno Central. Cubrimos la información de los pueblos por pequeños que sean.

En el Estudio General de Medios de octubre 2006 a mayo 2007, se demuestra que Ser y Canal Sur son las emisoras más escuchadas entre las 14:00 y 15:00 en Andalucía. El 32.08\% de la población andaluza consume, en esa franja horaria, radio generalista. Y, de estos oyentes, el 34,9\% escucha la Ser y el 22,75\% Canal Sur; son las dos emisoras más escuchadas seguidas de Onda Cero $(16,4 \%)$ y COPE $(1269 \%)$. En Málaga la audiencia se reparte de otra manera. El share de Ser es de $39,2 \%$ y el de Canal Sur de $12,6 \%$. En esta ciudad, Onda Cero y COPE ocupan el segundo y tercer lugar, respectivamente, en el ranking de audiencias.

\section{OBJETIVOS DEL ESTUDIO}

Los objetivos de este trabajo apuntan en una doble dirección. En primer lugar, este análisis de los contenidos de la radio local persigue conocer los temas que nutren la agenda malagueña y, en segundo lugar, determinar cómo se cubre la información política municipal. Asimismo, pretendemos:

1. Determinar el peso relativo de la capital y la provincia en los informativos radiofónicos locales. La diferente tipología de ambas emisoras hace considerar la hipótesis de que la cobertura de los pequeños municipios y las grandes ciudades Será muy diferente en ambas.

2. Conocer la temática y los actores que configuran los informativos de Canal Sur y Ser.

\footnotetext{
${ }^{4}$ Con respecto a los temas, el equipo de redacción manifiesta no tener líneas de prioridad. Se interesan por todos los temas en general, tanto de la capital como de la provincia. Para el Ayuntamiento hay una cobertura especial. Siempre hay una persona que se dedica a cubrir la información del Ayuntamiento de Málaga aunque no es siempre la misma persona.
} 
3. De forma más específica, dentro de la configuración temática, se estudia la cobertura de los temas políticos municipales en ambas emisoras. El análisis determinará cuál es la imagen del Ayuntamiento de Málaga, cuáles son los eventos políticos y los personajes que copan la agenda mediática de la capital andaluza.

4. Dentro del estudio de la información política, este trabajo plantea la hipótesis de que la adscripción ideológica de la cadena Ser determinará el sesgo de la cobertura de la actividad política municipal de forma más evidente de lo que sucede con la agenda de Canal Sur, una emisora pública dependiente de la Radio Televisión Andaluza.

En último lugar, el objetivo final, y casi totalizador, es determinar la imagen de Málaga que componen, a través de sus informaciones, estos medios locales. Si el espejo mediático ofrece una imagen realista o distorsionada. La selección de temas y el tratamiento de los mismos permiten estudiar los términos en los que se establece el debate público sobre la ciudad en materia política.

\subsection{Selección de la muestra y metodología}

Ser y Canal Sur emiten por las mañanas para todo su radio de acción -una nacional y la otra andaluz- pero desconectan alrededor de las 12:00 para difundir programas de tertulias y entrevistas sobre la actualidad malagueña que dan paso a los informativos ${ }^{5}$. Al medio día ambas realizan desconexiones territoriales. Este informativo de medio día es la unidad muestral de este estudio: Cadena Ser, de las 14:15 a las 14:30; Canal Sur, de las 14:45 a las 14:55.

El periodo analizado es de dos meses. La muestra ha quedado compuesta por los informativos desde el 10 de septiembre de 2007 hasta el 10 de noviembre del mismo año, emitidos de lunes a viernes, excluidos los días festivos. La contemplación de semanas naturales y el representativo periodo de tiempo abarcado para un estudio de esta naturaleza permite subsanar las distorsiones que etapas más cortas podían suponer. Esto es, un estudio diacrónico permite analizar cómo evolucionan las diferentes temáticas en la agenda mediática mientras que, contemplar un periodo de menor duración, podría mostrar que algunos asuntos centralizan la atención mediática en un determinado momento y no dejaría observar la construcción periodística de la realidad a medio plazo.

En mayo se celebraron elecciones municipales y en marzo de 2008 tuvieron lugar las autonómicas y generales. En este marco temporal, el periodo comprendido entre septiembre y noviembre es de alta intensidad política -tras el verano- y sortea las campañas electorales de ambos comicios. De esta forma, ya con un Ayuntamiento constituido y con cierto rodaje, se persigue que la muestra seleccionada se centre en la realidad de la ciudad de Málaga, sus instituciones y sus proyectos.

Con estas premisas se ha diseñado una ficha de análisis dividida en dos partes. En primer lugar, un apartado que se completa para todas las noticias comprendidas

\footnotetext{
${ }^{5}$ A las 12:03 Inmaculada Jabato presenta "De par en par" en Canal Sur. A las 12:20 comienza "Hoy por hoy Málaga" dirigido por Esther Luque.
} 
en el marco temporal. Se recoge si es primera noticia, el municipio o municipios a los que se refiere la noticia, a qué sección pertenece y, más concretamente, sobre qué tema trata. El análisis de los datos se inició concluidos los dos meses incluidos en la muestra así que se realizó una lista estimativa cerrada de temas para los más continuos, a partir de esa escucha, y se dejó una opción abierta de respuesta para otros asuntos.

La segunda parte de la ficha de análisis se dedica sólo a las noticias políticas. Se analizan las instituciones que protagonizan la información, todas aquellas que aparecen referidas sean políticas o no. Posteriormente se señala quién genera el titular. Se recoge si existe sesgo en el tratamiento del Ayuntamiento de Málaga, la Junta de Andalucía, la Diputación o el Gobierno en las piezas en las que se les menciona y, por último, se registra si hay declaraciones (hasta tres por noticia).

\section{LA AGENDA LOCAL EN LA RADIO MALAGUEÑA: ANÁLISIS}

Se han analizado un total de 70 informativos, entre ambas cadenas ${ }^{6}$. El número medio de noticias por día es de 8,2 para Ser (287 fue el total de ítems registrados para esta emisora) y 7,3 para Canal Sur (254 noticias). No debe olvidarse que el noticiero de Ser dura aproximadamente 15 minutos frente a los 10 de Canal Sur, por lo que cabe afirmar que la cadena andaluza dedica más tiempo a cada información: dura dos tercios del otro y tan sólo emite una noticia menos al día de media. El primero de los objetivos que se persigue con este trabajo es medir el peso de la capital y la provincia en estos informativos y conocer la temática que se asocia en los informativos a cada uno de estos escenarios.

Tabla 1. Peso de la capital y la provincia.

\begin{tabular}{|lrrr|}
\hline Emisora & Ámbito & \multicolumn{1}{c}{ Total } & $\%$ \\
\hline Ser & Sólo Málaga Capital & 123 & $42,90 \%$ \\
\hline Ser & Provincia & 164 & $57,10 \%$ \\
\hline Canal Sur & Sólo Málaga Capital & 94 & $37,00 \%$ \\
\hline Canal Sur & Provincia & 160 & $63,00 \%$
\end{tabular}

Fuente: Elaboración propia.

Esta tabla 1 muestra que en ambas emisoras la provincia parece tener un mayor peso cuantitativo. En Canal Sur es especialmente significativo porque, como se comentó, carece de emisoras comarcales. En Ser el porcentaje es algo más igualado aunque también prima la provincia.

Al considerar los municipios aisladamente, Málaga es, para ambas, el centro de acción ${ }^{7}$. En Ser es protagonista del 49,1\% de las piezas y en Canal Sur del 39,4\%. Ambas emisoras repiten un pódium muy similar en este apartado pues el segundo lugar es ocupado por la provincia en general y, en tercer lugar, por Marbella. Cabe

\footnotetext{
${ }^{6}$ La existencia de varias festividades nacionales en el periodo analizado hizo descender el número de piezas que a priori se calculó que se iba a incluir.
} 
mencionar que Canal Sur realiza una cobertura más diversificada en la que, por ejemplo, los pueblos pequeños, en conjunto, son protagonistas del $3,9 \%$ de sus noticias y Antequera y Torremolinos de 3,1\% cada uno. Así pues, en términos cuantitativos, de aquí se desprende que la provincia está más representada en Ser y Canal Sur que la capital pero que, si consideramos noticias que afectan a Málaga ciudad a la vez que a otras localidades, ésta es, sin duda, la protagonista de los informativos locales. Es ahora el momento de estudiar para qué temas y en qué secciones aparecen capital y provincia en los boletines radiofónicos, pues existe la hipótesis inicial de que una y otra tienen perfiles muy diferentes para las cadenas.

Con respecto a las secciones, la suma total de la muestra evidencia que política $(14,20 \%)$, sociedad (14\%) e infraestructuras e inversiones $(13,7 \%)$ son las tres más representadas. Sin embargo, una discriminación entre ambas cadenas muestra que es Ser la que sitúa a la política como primer tema de la agenda pues, para la cadena pública, es un tema secundario: representa el $19,20 \%$ de las noticias de Ser frente al $8,7 \%$ de Canal Sur. En ésta, la cultura y el arte completan el pódium ${ }^{8}$.

La contribución de la capital y la provincia a esta clasificación es muy desigual. Lejos de pensar que la aportación de noticias políticas de una y otra es proporcional, lo cierto es que la provincia aparece para sucesos y sociedad más que para política. Como se observa en este desglose, para Ser la política es el tema principal, de forma destacada, seguido por las infraestructuras e inversiones. La cadena andaluza invierte el orden. Para ambas, la cultura es el tercer tema en importancia en la ciudad ${ }^{9}$.

La cobertura de la provincia es considerablemente diferente. La emisora de Prisa evidencia que la política sigue siendo una de sus secciones destacadas pero, para estos municipios, la importancia es mucho menor. En Canal Sur, la cobertura de la actividad política en los pueblos tiene menos relevancia que los temas turísticos o sanitarios. Ello demuestra que el debate que se articula sobre la provincia minimiza el discurso político y se centra en otros temas con menor contenido cívico. La cultura, además, otro tema de indudable contenido modernizador, ocupa un lugar casi intrascendente en la provincia.

\footnotetext{
${ }^{7}$ La Tabla 1 alude a las noticias referidas sólo y exclusivamente a Málaga capital. Sin embargo, los datos ofrecidos en este párrafo dan más relevancia a Málaga porque también se ha considerado que había ciertas piezas en las que se aludía a la capital como cabeza de la información, y ello la hacía protagonista, pero no excluía a otros municipios de tomar parte de la misma. Por ejemplo: "El parlamentario malagueño Ildefonso del Olmo dejará la política tras las próximas elecciones", en Canal Sur, 9 de octubre; o algunas piezas referidas al acceso al aeropuerto desde Torremolinos.

${ }^{8}$ El pódium de cada emisora es el siguiente. En Ser, Política (19,2\%), Infraestructuras e inversiones $(13,6 \%)$ y Sociedad (12,5\%); en Canal Sur, Sociedad (15,7\%), Infraestructuras e inversiones $(13,8 \%)$ y Cultura y arte $(12,2 \%)$.

${ }^{9}$ Los porcentajes suman más del $100 \%$ porque cada noticia podía estar incluida en dos secciones. Los porcentajes están calculados sobre el total de noticias (541), el total de noticias en Ser (287) y el total de piezas en Canal Sur (254). Téngase esta anotación en consideración para el resto de variables en las que se haya admitido respuesta múltiple.
} 
Tabla 2. Sección de Málaga capital.

\begin{tabular}{|c|c|c|}
\hline Sección Málaga & $\%$ Ser & $\%$ CS \\
\hline Infraes. e Inver. & $22,00 \%$ & $22,30 \%$ \\
\hline Política & $25,20 \%$ & $16,00 \%$ \\
\hline Cultura & $20,30 \%$ & $21,30 \%$ \\
\hline Urbanismo & $15,40 \%$ & $10,60 \%$ \\
\hline Sociedad & $9,80 \%$ & $16,00 \%$ \\
\hline M. Ambiente y Agua & $8,90 \%$ & $5,30 \%$ \\
\hline Econ. Y Empresas & $4,10 \%$ & $7,40 \%$ \\
\hline Sucesos & $7,30 \%$ & $3,20 \%$ \\
\hline
\end{tabular}

Fuente: Elaboración propia.

Tabla 3. Sección de la provincia.

\begin{tabular}{|r|r|r|r|}
\hline Sección Provincia & $\%$ Ser & Sección Provincia & $\%$ CS \\
\hline Sucesos & $15,20 \%$ & Sociedad & $15,60 \%$ \\
\hline Sociedad & $14,60 \%$ & Sucesos & $13,80 \%$ \\
\hline Política & $14,60 \%$ & Medio Ambiente & $13,80 \%$ \\
\hline Medio Ambiente & $11,00 \%$ & Economía y Empr & $12,50 \%$ \\
\hline Economía y Empr & $11,00 \%$ & Policía y Justicia & $10,00 \%$ \\
\hline
\end{tabular}

Fuente: Elaboración propia.

Una vez estudiadas las secciones y su representación en la agenda de cada medio, el estudio profundiza en los temas que son tratados en ambas. La configuración temática sobre la capital, como se muestra en la tabla 4, tiene una coincidencia en la importancia concedida a la agenda cultural: ambas emisoras intentan cerrar sus informativos con alguna pieza sobre ello. Es cierto que su importancia cuantitativa es muy alta, pero hay que tener en cuenta que la duración de estas piezas y su situación en el informativo no es destacada.

Tabla 4. Temas sobre Málaga capital.

\begin{tabular}{|l|r|r|}
\hline \multicolumn{1}{c}{ Tema Málaga } & \% CS & \multicolumn{1}{c|}{ Tema Málaga } \\
\hline Agenda cultural & $12,80 \%$ Agenda cultural & $7,30 \%$ \\
\hline Inversiones & $7,40 \%$ Medio Ambiente & $6,50 \%$ \\
\hline Empresas & $6,40 \%$ Pleno municipal & $5,70 \%$ \\
\hline Palacio de Justicia & $5,40 \%$ CAC & $4,90 \%$ \\
\hline Congresos & $4,30 \%$ Inversiones & $4,90 \%$ \\
\hline Medio Ambiente & $3,20 \%$ Presupuestos ciudad & $4,90 \%$ \\
\hline Pleno municipal & $3,20 \%$ Empresas & $4,10 \%$ \\
\hline Presupuestos ciudad & $3,20 \%$ Palacio de Justicia & $4,10 \%$ \\
\hline Turismo y datos & $3,20 \%$ Metro & $3,60 \%$ \\
\hline Metro & $2,10 \%$ Aeropuerto & $2,40 \%$ \\
\hline Sucesos & $2,10 \%$ Málaga 2016 & $2,40 \%$ \\
\hline Problemas de limpieza & $2,10 \%$ Problemas de la vivienda & $2,40 \%$ \\
\hline Urbanismo & $2,10 \%$ Turismo y datos & $2,40 \%$ \\
\hline Abastecimiento de Agua & $2,10 \%$ Sucesos & $2,40 \%$ \\
\hline VPo & $2,10 \%$ Problemas de limpieza & $2,40 \%$ \\
\hline Agresiones escolares & $2,10 \%$ Urbanismo & $2,40 \%$ \\
\hline Laboral & $2,10 \%$ Abastecimiento de Agua & $1,60 \%$ \\
\hline Malos Tratos & $2,10 \%$ AVE & $1,60 \%$ \\
\hline Catedral & $2,10 \%$ Museo Picasso & $1,60 \%$ \\
\hline Tabacalera & $2,10 \%$ VPO & $1,60 \%$ \\
\hline
\end{tabular}

Fuente: Elaboración propia. 
Para la emisora pública, las inversiones y la actividad empresarial son los otros temas destacados. El cuarto lugar lo ocupa el debate sobre el Palacio de Justicia de Teatinos $^{10}$, seguido por los congresos que aquí se desarrollan. En líneas generales, observando la lista de temas, ofrece un discurso que elude las confrontaciones políticas. Ser entra de lleno en ellas: el pleno municipal, el CAC, el debate sobre presupuestos o el metro son tratados con asiduidad. La cadena de Prisa dedica una cobertura amplia a cada uno de los proyectos que se están acometiendo para modernizar la ciudad y es bastante frecuente que sean polémicas o cruce de declaraciones políticas las que originen estas noticias.

En Canal Sur estos proyectos son cubiertos cuando hay alguna novedad en el proyecto de las mismas, alguna nueva adjudicación o puntualmente si hay alguna discusión especialmente significativa. Paradigmático es el caso del CAC: en estas fechas se produce el segundo concurso para su adjudicación después de que la primera quedara desierta al ser desestimadas por el consistorio las propuestas de Unicaja y del anterior gestor, Fernando Francés. En Canal Sur la cobertura es prácticamente inexistente.

Ambas emisoras otorgan un trato muy destacado al medio ambiente. Se desarrollan en este periodo muchas iniciativas por parte de la Consejería de Medio Ambiente, de la Delegación de Innovación y el Área de Medio Ambiente del Ayuntamiento. El calentamiento global se ha convertido en un tema recurrente en la opinión pública y la radio malagueña se hace eco de ello.

En la provincia, como se observa en la siguiente tabla, la importancia de éste y los temas anteriormente destacados, es muy diferente. Los municipios malagueños aparecen en la radio local para sucesos (asesinatos, incendios, robos en sucursales bancarias, agresiones escolares, etc.). A pesar de que Canal Sur dedica una mayor cobertura a la provincia que a la capital y de que no tiene cabeceras comarcales, ésta suele aparecer para temas no políticos y ello reduce el ámbito de discurso público en los municipios malagueños. Los medios locales son el primer nivel en la socialización y creación de una conciencia ciudadana de la estructura mediática, si se elude la actualidad política en este entorno cercano, se anula su potencial como movilizador de la opinión pública.

Se puede observar la importancia de Marbella para la Ser pues el seguimiento de los juicios derivados de la operación Malaya y la constitución de su ayuntamiento, son claves en la agenda provincial de la misma. En la emisora pública, por su parte, las inversiones, los datos del sector turístico o la innovación empresarial son otros temas fundamentales que muestran que se quiere difundir una imagen dinámica de la provincia.

\footnotetext{
10 En estos meses se produjo el inicio de la mudanza de los órganos judiciales a las nuevas dependencias en Teatinos. A ello hay que añadir el problema de que la falta de aparcamientos derivó en un cruce de declaraciones entre Junta de Andalucía y Ayuntamiento de Málaga, que afirmó que no concedería la licencia de primera ocupación si no se solucionaba el problema. La consejera dijo que no dudaría en denunciar al Ayuntamiento de Málaga si impedía el traslado. Finalmente se comenzó la mudanza en su fecha, el 4 de octubre.
} 
Laura Teruel Rodríguez

Tabla 5. Tema sobre la provincia.

\begin{tabular}{|c|c|c|}
\hline Tema Málaga provincia & \% Ser Tema Málaga provincia & $\%$ CS \\
\hline Sucesos & $10,40 \%$ Sucesos & $13,10 \%$ \\
\hline Operación Malaya & $7,90 \%$ Inversiones & $6,90 \%$ \\
\hline Laboral & 5,50\% Operación Malaya & $5,00 \%$ \\
\hline Turismo y datos & $5,50 \%$ Turismo y datos & $5,00 \%$ \\
\hline AYMB constitución & 5,50\% Empresas & $5,00 \%$ \\
\hline Abastecimiento de Agua & 4,30\% Malos Tratos & $3,80 \%$ \\
\hline Inversiones & $4,30 \%$ PGOU & $3,80 \%$ \\
\hline Infraest sanitarias & 4,30\% Agenda cultural & $3,80 \%$ \\
\hline Congresos & 4,30\% Lengua Azul y ganadería & $3,80 \%$ \\
\hline Malos Tratos & 3,70\% Medio Ambiente & $3,80 \%$ \\
\hline AVE Valle Abdalajís & 3,00\% AVE Valle Abdalajís & $3,10 \%$ \\
\hline Droga & $3,00 \%$ Abastecimiento de Agua & $2,50 \%$ \\
\hline Medio Ambiente & 2,40\% Droga & $2,50 \%$ \\
\hline
\end{tabular}

Fuente: Elaboración propia.

\subsection{La información política local en la radio malagueña}

El tercero de los objetivos trazados para este trabajo profundizaba en la trascendencia de la política local en la radio malagueña. Para ello, en primer lugar, era necesario saber si la presencia de la capital iba ligada en estas emisoras a temas políticos: aunque en el caso de Ser es más abultada la diferencia, lo cierto es que así es.

Tabla 6. Tema político sobre Málaga.

\begin{tabular}{|c|c|c|}
\hline Tema Político & Ser $\%$ & $\% \mathrm{CS}$ \\
\hline SI & $67,50 \%$ & $54,30 \%$ \\
\hline NO & $32,50 \%$ & $45,70 \%$ \\
\hline TOTAL & $100 \%$ & $100 \%$ \\
\hline
\end{tabular}

Fuente: Elaboración propia.

Ya centrado en la capital, la política municipal cardina sobre el Ayuntamiento. Es para las dos emisoras, la institución protagonista de las noticias políticas sobre la ciudad: para Ser protagoniza el $68,7 \%$ de las piezas sobre la capital y, para Canal Sur, el $72,5 \%$. Es algo, sin duda, comprensible.

Ambas cadenas coinciden también en que la segunda instancia en la política malagueña es la Junta de Andalucía (Ser 51,8\%, Canal Sur 66,75\%). La inauguración de infraestructuras sanitarias, del Palacio de Justicia, de las instalaciones de reciclaje y tratamiento de residuos, la aprobación de los presupuestos, el debate sobre el PGOU y el POT, etc., explican esta relevancia.

En los restantes puestos de la clasificación, existe discordancia. Ser sitúa, en tercer lugar, al Gobierno central (30.1\%). Para la emisora pública, el tercer lugar es ocupado por otras instancias. Como se ha explicado, la carga política de Canal Sur es menor pues suele obviar las discusiones partidistas que predominan en Ser. Además de ello, se caracteriza por una amplia cobertura del sector laboral, empresarial 
y turístico; ello explica que un $41,2 \%$ de sus noticias políticas sobre la ciudad estén protagonizadas por el conglomerado formado por agentes como los sindicatos, la Universidad de Málaga, la Policía, Facua, Consorcio de Transportes del Área Metropolitana, Unicaja, etc.

Más concretamente, establecidas ya las coordenadas locales, autonómicas y nacionales que enmarcan la información sobre la ciudad, se profundizó sobre qué áreas de estas instancias articulan el debate público. Las emisoras sólo están de acuerdo en que la alcaldía es el primer interlocutor de la ciudad. También existe acuerdo sobre la importancia del Ministerio de Fomento pero, antes de éste, la Ser entiende que el PSOE ${ }^{11}$ es el segundo participante en la escena municipal.

Tabla 7. Área de las instituciones políticas sobre Málaga capital.

\begin{tabular}{|c|c|c|}
\hline Área de la Institución & Área de la Institución & $\% \mathrm{CS}$ \\
\hline Ay Alcaldía & $26,50 \%$ Ay Alcaldía & $21,60 \%$ \\
\hline PSOE & $20,50 \%$ Min Fomento & $19,60 \%$ \\
\hline Min Fomento & 16,90\% Diputación & $13,70 \%$ \\
\hline IU & $16,90 \%$ IU & $13,70 \%$ \\
\hline PP & 15,70\% Ayuntamiento Málaga & $11,80 \%$ \\
\hline Ay Cultura & 9,60\% Junta de Andalucía & $11,80 \%$ \\
\hline
\end{tabular}

Fuente: Elaboración propia.

Ser sigue haciendo patente su focalización en temas políticos pues los tres partidos con representación local son agentes destacados en sus noticias. Dentro del Ayuntamiento, Cultura, Urbanismo, Economía y Empleo y Medio Ambiente son las concejalías con más repercusión. En la cadena andaluza los protagonismos estás más repartidos por lo que priman las noticias que hablan sobre el Ayuntamiento en general, sin especificar área. En el caso de la Junta sucede lo contrario: Canal Sur realiza una cobertura más constante de varias de sus consejerías, destacando la de Salud.

Resulta muy interesante, para este trabajo, no sólo estudiar quién protagoniza la información local sino quién la genera, quiénes son los elementos activos y pasivos en el debate municipal. Los datos muestran ciertos paralelismos pero, sobre todo, interesantes divergencias con respecto a la tabla anterior. La alcaldía genera el 17,6\% de las noticias en Canal Sur y en Ser 18,1\%. Pero, en ésta última, el segundo lugar lo ocupan otros emisores. Bajo este epígrafe se engloban las noticias emitidas por FACUA, Policía, sindicatos, etc., pero, sobre todo, las que la propia emisora obtiene y difunde ${ }^{12}$. Así pues, la cadena de PRISA tiene un alto nivel de mediación en su propia agenda.

\footnotetext{
${ }^{11}$ La categoría PSOE abarca tanto al partido en el Ayuntamiento como en el Parlamento Andaluz o el Gobierno central; todos los miembros y portavoces que no hablan como responsables de un ministerio o consejería pues para ellos hay una categoría específica.

12 Por ejemplo: "Más del 30\% de los vecinos de la capital malagueña soporta un índice de ruidos superiores a los recomendados por la OMS", en Ser, 19 de octubre. "Lo que también acumula retraso es el traslado de LIMASA a sus nuevas instalaciones en los Ruices", en Ser, 17 de septiembre. "Nuevo desencuentro entre la Junta y el Ayuntamiento por la inauguración del palacio de justicia”, en Ser, 27 de septiembre.
} 
El Ministerio de Fomento es el segundo agente en Canal Sur $(15,7 \%)$ y el tercero en $\operatorname{Ser}(7,2 \%)$, donde empata con las representaciones locales y autonómicas de Izquierda Unida. Más concretamente, se ha estudiado quiénes declaran en estas emisoras y ello ha reflejado un paralelismo con las áreas de las instituciones políticas anteriormente reflejadas. La tabla 8 muestra que el alcalde es el protagonista absoluto en ambas cadenas. Dentro de esta institución es sobre todo Canal Sur quién da más voz a los líderes de las restantes formaciones políticas; en Ser también tienen cabida sus declaraciones pero, ciertamente, son Miguel Briones (concejal de Cultura) y Elías Bendodo (concejal de Juventud y Deportes) los representantes del gobierno local que más cubre la emisora.

Entre ellos, de forma invariable, la ministra de Fomento. Magdalena Álvarez y su ministerio son agentes de primer nivel en el panorama local. Es un protagonismo muy destacado para una ministra en un escenario local; sin duda, ello obedece no sólo al gran número de infraestructuras que se están ejecutando en Málaga sino a que es la número 1 del PSOE por Málaga en el Congreso. Es la verdadera oponente del alcalde, en cuanto a relevancia y apariciones, para los medios analizados pues, de la oposición, el protagonismo de Marisa Bustindy o Pedro Moreno Brenes es bastante menor ${ }^{13}$.

Tabla 8. Declaraciones sobre Málaga capital.

\begin{tabular}{|c|c|c|c|}
\hline Declaraciones & $\%$ Ser & Declaraciones & $\% \mathrm{CS}$ \\
\hline AM Francisco de la Torre & $21,70 \%$ & AM Francisco de la Torre & $25,50 \%$ \\
\hline AM Miguel Briones & $9,60 \%$ & Gob Magdalena Álvarez & $13,70 \%$ \\
\hline Gob Magdalena Álvarez & $9,60 \%$ & AM Elías Bendodo & $11,80 \%$ \\
\hline AM Elías Bendodo & $7,20 \%$ & AMPSOE Marisa Bustinduy & $9,80 \%$ \\
\hline Otro & $7,20 \%$ & Ay-IU Pedro Moreno Brenes & $9,80 \%$ \\
\hline Ay-IU Pedro Moreno Brenes & $6,00 \%$ & DPM Salvador Pendón & $9,80 \%$ \\
\hline JA Concepción Gutiérrez & $6,00 \%$ & JA María Jesús Montero & $7,80 \%$ \\
\hline AM Araceli González & $4,80 \%$ & JAD María Gámez & $7,80 \%$ \\
\hline AM Carolina España & $4,80 \%$ & Otro & $7,80 \%$ \\
\hline AM Manuel Díaz, AMPSOE Marisa & $4,80 \%$ & AM Miguel Briones, JA & $5,90 \%$ \\
\hline Bustinduy JA María Jesús Montero, & & Concepción Gutiérrez, PA & \\
\hline PA Esperanza Oña, PP Celia & & Antonio Romero, PA Esperanza & \\
\hline Villalobos & & Oña, AM Araceli González & \\
\hline
\end{tabular}

Fuente: Elaboración propia.

Los medios de comunicación seleccionan los acontecimientos, las declaraciones y los enfoques y, con ello, interpretan la actualidad y la dotan de sesgos. Como se planteaba en el cuarto objetivo de esta investigación se persigue determinar los ses-

13 En términos sociales, es muy positivo destacar que la paridad está cercana en la política malagueña pues el porcentaje de declaraciones de hombres y mujeres se salda con datos cercanos a la igualdad: los diez primeros puestos revelan que el $44,4 \%$ de declaraciones son de mujeres en Ser frente al $49,3 \%$ masculino, y $56,8 \%$ femenino en Canal Sur frente al $68,7 \%$. 
gos que ambas emisoras imprimen a la cobertura de la información política municipal. Por el número de piezas, sólo es contundente el resultado obtenido sobre el Ayuntamiento de Málaga.

Tabla 9. Sesgo en la información sobre el Ayuntamiento.

\begin{tabular}{|l|c|c|c|c|c|c|}
\hline \multicolumn{2}{|c|}{$\begin{array}{c}\text { Sesgo información AYM } \\
\text { Negativo }\end{array}$} & Total & Ser & $\%$ Ser & Canal Sur & $\%$ CS \\
\hline Neutro & 42 & 28 & $43,80 \%$ & 14 & $37,80 \%$ \\
\hline Positivo & 37 & 27 & $42,20 \%$ & 10 & $27,00 \%$ \\
\hline & \multicolumn{1}{|c|}{ Total } & 22 & 9 & $14,10 \%$ & 13 & $35,10 \%$ \\
\hline
\end{tabular}

Fuente: Elaboración propia.

Los datos demuestran que tanto en Ser como en Canal Sur predomina un tono negativo hacia el Ayuntamiento de Málaga en la información que le alude. No es un sesgo absoluto, puesto que en Canal Sur la cobertura está polarizada entre lo positivo y lo negativo y en Ser la tendencia es neutra-negativa. Pero lo cierto es que en esta última es verdaderamente escaso el número de atribuciones positivas al consistorio. Con respecto a la Junta de Andalucía, los porcentajes no son tan elocuentes. En ambas emisoras predomina la neutralidad en la cobertura del ente autonómico (60,6\% en Ser / 41,9\% en Canal Sur) pero en Canal Sur tiene una tendencia claramente positiva $(41,9 \%)$ y en la emisora de Prisa negativa $(24,2 \%)$. La clasificación sobre el Gobierno es similar a la de la Junta de Andalucía.

Estos datos suscitan varias argumentaciones, pues la escucha de los informativos, antes de su codificación, hacía pensar que el sesgo negativo del Ayuntamiento de Málaga y el positivo del Gobierno serían mucho más visibles. Por una parte, el tratamiento de la información municipal: la Ser nunca omite detalles sobre retrasos, polémicas, hacer oídos sordos a peticiones de la oposición o de ciertos colectivos, etc. El análisis muestra que lo que sucede es que, cuando una noticia es negativa para el Ayuntamiento de Málaga, lo es profundamente, y prima la calidad de noticias malas a la cantidad ${ }^{14}$.

Por otra parte, la información sobre las infraestructuras relativas a la Junta de Andalucía y al Gobierno, sobre todo a Fomento, no siempre se realiza recordando la titularidad de éstos y, en muchas ocasiones, la noticia consiste tan sólo en anunciar que empieza la fase de concurso, que termina, para cuándo se prevé su finalización, el estado de las obras, etc. Es una forma de mantener estos temas en la agenda pública y de que, quienes sepan cuál es la institución encargada de su desarrollo, refuerce su conocimiento. En general, las múltiples declaraciones de Mag-

\footnotetext{
14 Por ejemplo, en "El casco histórico de la ciudad está considerado como de los más ruinosos de España" (en Canal Sur, 19 de septiembre) se narraba con detalles el estado de dejadez de los edificios por parte del Consistorio. En "El gobierno central responsabiliza al Ayuntamiento del retraso en la construcción de los desvíos de la autovía en la barriada de Tiro Pichón" (en Canal Sur, 30 de octubre), sin réplica local. En "El equipo de gobierno de PP aprueba hoy la subida del agua en Málaga con la oposición de PSOE e IU" (en Ser, 12 de septiembre), se explica que la tasa sube más que el IPC y se replica desde el medio la respuesta de la concejala.
} 
dalena Álvarez se realizan en estos términos y las emisoras no suele mediar tanto en estos contenidos como con el Ayuntamiento de Málaga. En el caso de Canal Sur, de titularidad pública, el sesgo hacia la Junta de Andalucía no es muy destacado porque sucede lo mismo.

\section{CONCLUSIONES}

En el apartado anterior se han ido exponiendo las conclusiones más inmediatas sobre el análisis de los datos. A continuación se exponen los resultados finales del trabajo y se evalúa la consecución de los objetivos trazados.

Existe un primer aspecto positivo, ambos medios reflejan la Málaga que crece, la que está en pleno desarrollo. El capítulo de infraestructuras e inversiones es destacado tanto para la capital como para la provincia. En este sentido, es incuestionable que se refleja la imagen más dinámica de ambas. También es positivo el valor concedido a la cultura, a las infraestructuras que se están desarrollando, a los congresos que acogen capital y provincia.

Pero, en otros aspectos, la provincia aparece desdibujada en los informativos radiofónicos locales. Su reflejo en la agenda se basa en sucesos y sociedad y no tanto en el debate político continuo. Ello, sin embargo, da cabida a más interlocutores de diferente procedencia, especialmente en Canal Sur.

La provincia tiene claro que Málaga es la sede administrativa, el motor de crecimiento. La ciudad ve que la provincia carece de importancia política, sólo aparece de forma discontinua para este aspecto frente a la primacía de los sucesos. Aunque no debe olvidarse de que hay comarcas con desconexiones propias de Ser y de que Canal Sur, después de los sucesos, deja aflorar las inversiones, la situación laboral y las iniciativas empresariales de la provincia. Ello deja una puerta abierta a que Ser trate esos temas en sus diferentes emisoras y de que la cadena pública no haga en la provincia algo diferente de lo que hace en la capital: eludir la confrontación política.

Existe una diferencia significativa en la cobertura de la cadena pública con respecto a la privada. Canal Sur obvia el debate político, la confrontación institucional. Cabe preguntarse si lo hace porque ello no aporta nada sustancial a la opinión pública o porque, al tratarse de un ente público, estima que no le reporta nada adentrarse en este estilo. Por el lado contrario, Ser profundiza ampliamente en los desencuentros institucionales. Se han contabilizado bastantes piezas en las que el acontecimiento desencadenante es la pura discusión y, lejos del conteo, el análisis cualitativo es contundente al respecto. Toca, por tanto, preguntarse si mostrar un escenario tan polarizado es beneficioso para enganchar a la ciudadanía a la información política local. Recientes teorías afirman que ofrecer información política, de alto contenido crítico, no desanima a los ciudadanos sino que, por el contrario, se desarrolla un círculo virtuoso que anima a los más interesados a tomar parte:

Los más versados en cuestiones políticas, los que más confían y los más participativos, se muestran más proclives a atender la cobertura informativa sobre asuntos públicos. Y aquellos más atentos a la cobertura sobre asuntos públicos, se comprometen más con la vida pública (Norris, 2000: 317). 
Autoras como Rodero y Sánchez insisten en que el grado de politización de la radio local le está haciendo perder credibilidad y la aleja de renovar su audiencia. Situar al periodismo político local de Ser entre ambos extremos es, ciertamente, una cuestión nada sencilla. Por una parte, su audiencia crece año tras año. Por la otra, la sociedad cada vez responsabiliza más a los medios de crispar el debate tanto como los políticos.

En el ámbito institucional, hay que destacar la importancia del Ayuntamiento de Málaga en ambas cadenas. A pesar de que es el protagonista absoluto de las informaciones sobre la ciudad, no controla la agenda de los medios pues, por una parte, aunque declara mucho, es objeto de un sesgo claramente negativo por parte de ambas emisoras. Y, por la otra, no logra incluir en la agenda los temas que más le convienen casi nunca: basta con comprobar que rara vez la noticia viene a anunciar simplemente una medida adoptada por el equipo de gobierno. Las emisoras median, interpretan, seleccionan el enfoque y recurren a otras fuentes por lo que, en estas dos cadenas de signo contrario al del Ayuntamiento, casi nunca logra el altavoz mediático que les gustaría.

El periodismo desarrollado en Ser es más abogado, redacta las noticias para resaltar su importancia y les imprime un enfoque político. Ciertamente el ritmo del informativo está más dominado por los conocimientos de los profesionales y los temas de fondo que por la simple actualidad informativa porque, en muchas ocasiones, se ha querido ampliar alguna noticia en la prensa local digital y no había aparecido: Ser guía su propia agenda. Canal Sur, por su parte, desarrolla un periodismo difusor, en el que se pretende narrar los hechos sin que exista un enfoque ideológico o contextual por parte de la emisora (Canel y Sánchez-Aranda, 1999). Y ciertamente así es, en tanto no existe análisis de fondo ni continuidad en las noticias de largo recorrido.

Sin duda, el trabajo de la emisora pública permite un acceso más democrático a la radio. Al tratarse muchos temas empresariales, turísticos o laborales, se da voz a agentes diversos. Ser media más en la escena política y Canal Sur da más voz a los colectivos pero no tiene tanto protagonismo en el debate público como la cadena privada.

Por último, como se planteó al comienzo de este trabajo, cabe concluir que ambas emisoras proponen un acercamiento muy diferente al potencial reconocimiento de la identidad colectiva de la que emergen. Coinciden en la primacía de la capital, en el papel secundario y con menor peso político de la provincia; coinciden en reflejar las señas de identidad local y en mostrar que Málaga está en indudable cambio. Sin duda, se convierten para un foro de debate sobre lo próximo pero existe una contaminación de la situación política nacional en tanto la politización entre administraciones, a escala local, es visible e, incluso, ensalzado por Ser y escenificado por Canal Sur con una imagen positiva de las instituciones gobernadas por socialistas pero, de forma más destacada, por pasar de puntilla por las polémicas interpartidistas, algo, ciertamente, que no era previsible tratándose de una emisora pública española. 


\section{REFERENCIAS BIBLIOGRÁFICAS}

BLANCO CASTILLA, E. "La imagen de Málaga a través de los medios. Influencia del caso Malaya y de la delincuencia organizada", en La Universidad y nuestros mayores. Málaga: Servicio de Publicaciones, 2007.

CANEL, M. J.; SÁNCHEZ -ARANDA, J. J. (1999), en "La influencia de las actitudes profesionales del periodista español en las noticias". Anàlisi (1999), n 23. Disponible en http://ddd.uab.es/pub/analisi/02112175n23p151.pdf [Consulta: 18 de enero de 2008].

GUERRERO SERÓN, C. Los andaluces y los medios de comunicación. Hábitos y características de las audiencias. Sevilla: Junta de Andalucía, Consejería de la Presidencia, 1995.

GUTIÉRREZ, M. y HUERTAS, A. (2003), "La programación de las radios generalistas en España". Zer, n 15, pp.117-135.

HALLIN D.C.; MANCINI, P. Comparing Media Systems; Three Models of Media and Politics. Cambridge: University Press, 2003.

LÓPEZ, X. et ali. "El valor social de la información de proximidad". Revista Latina de Comunicación Social (1998), $n^{\circ} 7$. Disponible en http://www.ull.es/publicaciones/latina/a/68xose.htm [Consulta: 23 de enero de 2008].

LÓPEZ GARCíA, X. (2000):" La comunicación del futuro se escribe con $L$ de local". Revista Latina de Comunicación Social (2000), $\mathrm{n}^{\circ} 34$. Disponible en http://www.ull.es/publicaciones/latina/aa2000kjl/w34oc/41xose.htm [Consulta: 20 de enero de 2008].

MERLI, J. "A question of credibility". Disponible en http://www.broadcastingcable.com/article/CA14058.html [Consulta: 18 de enero de 2008].

NORRIS, P. A Virtuous Circle: Political Communication in Post-Industrial Societies. New York: Cambridge Press, 2000.

RODERO ANTÓN, E.; SÁNCHEZ SERRANO, Ch. "Radiografía de la radio en España". Revista Latina de Comunicación Social (2007), $\mathrm{n}^{\circ}$ 62.Disponible en http://www.ull.es/publicaciones/latina/200714RoderoySanchez.htm [Consulta: 17 de diciembre de 2007]. 


\section{Breve semblanza de la autora}

Laura Teruel Rodríguez se doctoró en Periodismo en 2006 con una tesis sobre el tratamiento de la política en la prensa nacional. Desde entonces, trabaja en los contenidos políticos en el periodismo y lo ha combinado con trabajos sobre la imagen de los andaluces en los medios. Hasta 2007 ha sido becaria de Formación de Profesorado Universitario en el Departamento de Periodismo de la Universidad de Málaga. Actualmente es miembro de la Cátedra UNESCO de Comunicación de la UMA, donde colabora en la coordinación de las Acciones UNESCO y el Observatorio de Libertad de Expresión, y del proyecto de investigación Proyecto "Nuevos Escenarios en la investigación aplicada al estudio del sistema de medios" I+D SEJ 2006-14561/soci, del Ministerio de Educación.

(Recibido el 31-01-08, aceptado el 29-04-08) 